\title{
Cervical Sympathetic and Stellate Ganglion Blocks
}

\author{
Mazin Elias, MD
}

Sympathetic blocks in the cervical and upper thoracic region are commonly used techniques for a variety of diagnostic, therapeutic and prognostic purposes. Stellate ganglion block is the common nomenclature utilized, however, stellate ganglion is present in only $80 \%$ of the population, thus, either lower cervical sympathetic block or upper thoracic sympathetic block is an appropriate term.

The cervical sympathetic ganglia are identified as the superior, middle, intermediate and the inferior cervical sympathetic ganglion. The superior cervical ganglia are approximately 3 to $5 \mathrm{~cm}$ in length and situated on the longus capitus muscle anterior to the transverse process of the second, third, and rarely the fourth cervical vertebrae; the middle cervical ganglia are the smallest of the cervical ganglia situated on the longus colli muscle, anterior to the base of the transverse process of the sixth vertebrae; and the intermediate cervical ganglia which are more consistent in position and are located on the medial side of the vertebral artery. The inferior cervical ganglia, when present, are located on the transverse process of the $\mathrm{C} 7$ vertebrae, whereas the first thoracic ganglia are situated in front of the neck of the first rib. In $70 \%$ to $80 \%$ of the population they are fused together forming the stellate ganglion.

Stellate ganglion block or lower cervical sympathetic block has been advocated for both diagnostic, therapeutic, and prognostic purposes for a variety of conditions. Even though multiple techniques are advocated in performing this block, fluoroscopically guided sympathetic blocks are more appropriate. Complications of stellate ganglion block include complications related to the technique, infection, and pharmacological complications related to the drugs utilized.

Cervical sympathetic or stellate ganglion block is a very commonly performed procedure. If performed correctly, this can provide good therapeutic, prognostic, and diagnostic values.

Keywords: Cervical sympathetic chain, stellate ganglion, cervical sympathetic block, stellate ganglion block, Horner's syndrome, post sympathetectomy neuralgia.
Blockade of the sympathetic chain/ganglia in the cervical and lower cervical and upper thoracic region is incorrectly referred to as stellate ganglion block. This is a commonly used technique for a variety of diagnostic, therapeutic and prognostic purposes. The stellate ganglion is present in only $80 \%$ of the population (1-3), so a more correct term should be either lower cervical sympathetic block or upper thoracic sympathetic block (cervicothoracic sympathetic trunk/block).

When a large volume of local anesthetic is used during the block, a wide lesion is produced and a cervical thoracic sympathectomy will result. The understanding of

From The Medical College of Wisconsin, Milwaukee, Wisconsin. Dr. Elias is associate professor and director of Pain Clinic, Milwaukee, WI. Address correspondence: M. Elias, MD, 9200 W. Wisconsin Ave., Milwaukee, Wisconsin 53226 these terms is very important, as well as the anatomy, to obtain an anatomically precise and pathophysiologically correct neural blockade.

\section{ANATOMICAL CONSIDERATIONS}

Understanding the anatomy of the cervical/thoracic sympathetic chain and ganglia is vital for precise diagnostic and therapeutic neural blockade, and to avoid unnecessary neuroablative procedures (4-6).

The peripheral sympathetic nerve supply to the head and neck is derived from preganglionic neurons having cell bodies located in the anterior lateral horn of the first and second thoracic spinal cord segment $(1,2)$. The axon will then pass via the anterior roots of the same spinal nerve level through the rami communicantes to join the upper cervical sympathetic ganglia. These cervical ganglia are identified as the superior, middle, intermediate, and inferior cervical sympathetic ganglia $(1,2)$. In only 
$80 \%$ of the population, the inferior cervical ganglia and the first thoracic ganglia fuse together to form the stellate ganglia (1-3). From these ganglia the postganglionic axons will pass upward along the internal/external carotid and vertebral arteries to the structures within the cranium. The axon may also join the grey rami communicantes; the latter will join the cervical nerve supply to the neck and the upper extremity (the cervical portion of the brachial plexus).

Four cervical sympathetic ganglia are anatomically identified bilaterally in most individuals. The upper three cervical sympathetic ganglia will be discussed briefly here, as they contribute very little to the practice of pain management.

The superior cervical ganglia are 3 to $5 \mathrm{~cm}$ in length and situated on the longus capitis muscle anterior to the transverse process of the second and third, rarely, the fourth, cervical vertebrae. These ganglia are believed to be the result of the fusion of four segmental ganglia corresponding to the upper four cervical nerves. This ganglion supplies branches to the internal carotid and jugular nerves and also gray rami communicantes to the second, third and sometimes the fourth cervical Somatic nerve and also contributes to the superior cervical cardiac nerve (1-3).

The middle cervical ganglia are the smallest of the cervical ganglias; they lie on the longest colli muscle, anterior to the base of the transverse process of C6 vertebra. They supply rami communicantes to the fifth, sixth and sometimes the fourth cervical nerves and supply branches to the carotid artery and carotid plexus.

The intermediate cervical ganglia are more consistent in position than the middle ganglia. They are located on the medial side of the vertebral artery in the front of the eighth cervical nerve. They have neural connection with the inferior cervical ganglia (or the stellate ganglia in $80 \%$ of the population in whom the first thoracic ganglia and the inferior cervical ganglia are fused). They are also connected to the ansa subclavia, which loops around the subclavian vessels. The ansa subclavia originates from the stellate ganglion and sends neural branches to the vertebral artery and the subclavian plexus.

Some of the sympathetic fibers bypass these three ganglia through the vertebral artery to supply some of the intraand extracranial structures. Thus, blockade of these ganglia produces inconsistent and incomplete sympathetic blockade to the head and neck structures. This, plus the fact that these ganglia are in close proximity to important structures (the carotid artery) and have inconsistent anatomical position makes them of little value in the management of sympathetically related disorders in the head and neck. Some authors have described the neural blockade of the superior and the intermediate cervical ganglia; however, these procedures will not be discussed due to their inefficiency in producing complete sympathetic blockade.

The inferior cervical ganglia when present as distinct structures are located on the transverse process of $\mathrm{C} 7$ vertebra. The first thoracic ganglia lie in the front of the neck of the first rib. When they are fused together, they form the stellate ganglia (in $70 \%$ to $80 \%$ of the population) (1-5). The stellate ganglion is oval in shape and measures $2.5 \mathrm{~cm}$ long, $1 \mathrm{~cm}$ wide and $.5 \mathrm{~cm}$ thick and is usually located behind the subclavian artery in the front of the first rib. The shape, however, and the position vary even in the same subject $(4,5)$. The stellate ganglion lies lateral and posterior to the lateral edge of the longus colli muscle as confirmed by autopsy and magnetic resonance imaging (MRI) result $(1-4,7)$. Since all the sympathic flow to the head and neck structures either synapse here at the sympathic ganglia, at the stellate ganglia, or pass through it to the more cephalic sympathetic ganglia, stellate ganglia block provides a more complete sympathetic denervation of the head and neck (2-5).

Stellate ganglia are separated by loose areolar tissue from the posterior osseous structures; which facilitates the spread of local anesthetic when deposited near the ganglia (2-4). Thus, this also explains the spread of local anesthetic to nearby structures, which are not related to the sympathetic ganglia (brachial plexus) resulting in false-positive response to stellate ganglion block. Posterior to the stellate ganglion lies the neck of the first rib and the transverse process of $\mathrm{C} 7$ vertebra, with the prevertebral fascia and the interspace fascia in between these two structures $(2,3)$.

The stellate ganglion lies on the longus colli muscle. Scalene groups of muscles and the brachial plexus are located laterally (2-4), which contributes to the spread of local anesthetic to the brachial plexus, producing somatic block rather than sympathetic block and contributing to a negative diagnostic test, especially if large volume of local anesthetic is used (4-6).

The ansa subclavia is a branch of the stellate ganglion that loops around the subclavian artery and reconnects 
again with the intermediate cervical sympathetic ganglia. The inferior cervical cardiac nerves arise from the medial aspect of the stellate ganglia and ansa subclavia and descend along the subclavian artery to join the cardiac plexus (this explains the cardiac effect of stellate ganglion block, including the bradycardia).

All the sympathetic nerves that supply the head and neck and most of those that supply the upper extremity traverse through the stellate ganglion. Thus, stellate ganglion block produces a more complete sympathetic denervation to the head and neck structures. However, there are a significant number of individuals in whom the intrathoracic somatic branches from the second thoracic spinal nerve join the first thoracic spinal nerve. This intrathoracic branch is joined by the gray rami communicantes carrying the sympathetic fibers that arise from the second thoracic sympathetic ganglia and possibly the T3 ganglia as well. These fibers join the lower part of the brachial plexus without passing through the stellate ganglia. This explains the incomplete sympathetic blockade of the upper extremities in a pure stellate ganglion block. A less common variety also exists that branches from the third thoracic spinal nerve and bypasses the stellate ganglion to the second spinal nerve. This second intrathoracic nerve also contains sympathetic fibers that arise from the third thoracic ganglia. This joins the second thoracic spinal nerve in proximity to the branch that later sends the first thoracic nerve. These anomalous pathways are referred to as the Kuntz's nerves. These anomalous pathways bypass the stellate ganglia and so pure stellate ganglion block will not produce complete sympathetic denervation of the upper extremity. As the sympathetic supply to the upper limb passes through the second and occasionally the third thoracic ganglia, these are the key relay stations which have to be blocked to produce sympathetic blockade for the upper extremity.

It is very important to understand the anatomical structure of the sympathetic ganglia, as a failure to do so can contribute to either false-positive or false-negative test results during sympathetic blockade. This can also contribute to the failure of some neuroablative procedures following what was considered to be a successful diagnostic stellate ganglion block (8-11).

\section{INDICATIONS}

Stellate ganglion/lower cervical sympathetic block has been advocated for diagnostic, therapeutic and prognostic purposes for a variety of conditions, including:
Chronic regional pain syndrome types I and II to the upper extremities (CRPS I and II) $(2,3$, 9-14);

Vascular insufficiency/occlusive vascular disorders, both acute and chronic, of the upper extremities, including Raynaud's disease, intra-arterial embolization and vasospasm following accidental intra-arterial injection of a drug $(2,3$, 10-13). Some also consider stellate ganglion block for vascular disease unsuitable for vascular reconstructive surgery, and for improvement of the blood flow following surgical graft or other vascular surgery $(13,15)$;

- Poor lymphatic drainage and local edema of the upper extremity following mastectomy $(2,15)$; Postherpetic neuralgia $(2,3,12,13,15)$;

Phantom pain $(2,13,15)$;

CRPS of the breast and pain following mastectomy $(15,17)$;

Quinine poisoning (2, 12, 13);

Sudden hearing loss and tinnitus (13, 18-21); Hyperhidrosis of the upper extremity $(9-11,13)$; Cardiac arrhythmias including; Jervell/Lang/ Nielson Syndrome and Ramano/Ward (idiopathic long Q-T syndrome), ischemic cardiac pain (2, 13-15, 22);

- Bell's palsy and a variety of orofacial pain syndromes, including neuropathic orofacial pain, and trigeminal neuralgia. (13, 15, 23-25);

Vascular headache, including cluster and migraine headaches and sympathetically maintained headaches (25-27); stellate ganglion block can aggravate migraine headaches in certain conditions and more appropriate sphenopalatine ganglion block probably should be performed (26); and

- Neuropathic pain syndromes in cancer pain (13, $15)$.

Stellate ganglion block has also been recommended for improving the blood flow to the cranium for angiography and following stroke/cerebrovascular accident (13) and hyperhidrosis to the upper extremities. $(10,13,15)$.

\section{TECHNICAL CONSIDERATIONS}

Multiple approaches have been used to localize the lower cervical sympathetic ganglia/stellate ganglia. These include ultrasound (27), MRI $(4,5,7)$ and/or plain fluoroscopic guidance (28). The classical approach, ie, blind technique, will be described here for academic interest. 
The author does not recommend this technique (blind technique) because the final position of the needle and the spread of the local anesthetic agent are unpredictable (47). Intravascular injection is reported despite a negative aspiration. Hogan and colleagues $(4,5)$ have reported the spread of the local anesthetic agent during sympathetic blockade to be anterior to the position of the stellate ganglia/cervical sympathetic ganglia. Thus fluoroscopic-guided stellate ganglia block is more appropriate to provide any diagnostic evaluation. However, for therapeutic purposes using a large volume of local anesthetic is acceptable. Thus a fluoroscopic-, MRI- or ultra-soundguided stellate ganglia block would be more appropriate and could provide diagnostic, therapeutic and possible prognostic value. The prognostic evaluation is very important, as it can determine whether the patient should proceed with a neuroablative procedure to the stellate ganglia or the lower cervical sympathetic ganglia block.

The paratracheal or anterior approach for the sympathetic blockade is performed with the patient in the supine position with the head slightly extended on a pillow to stretch the esophagus away from the transverse process. The landmark should be the Chassaignac's tubercle, which lies against the cricoid cartilage, which is the surface marking. This tubercle feels hard like a marble. Using two fingers, the sternocleidomastoid muscle can be retracted laterally; which will pull the internal carotid artery and the internal jugular vein away from the site of the insertion of the needle. The needle then is inserted between the cricoid cartilage and the fingers. The needle passes perpendicular to the skin and through the deep tissue until contact is made with the C6 tubercle. The depth varies from person to person. The needle is withdrawn a few millimeters away from the periosteum of the C6 transverse process and then, after negative aspiration tests for blood and CSF, $1 \mathrm{~mL}$ of the local anesthetic agent should be injected while observing for signs of intravascular or intrathecal injection. A negative aspiration doesn't exclude intravascular or intrathecal injection. Using fluoroscopy, the spread of the contrast can rule out intravascular, epidural and intrathecal injection. The patient should be advised prior to the block that he should refrain from swallowing and talking, as these can interfere with needle positioning. For communication, the patient can use his hand or fingers to signal to the operator if any changes are needed or if he requires any additional help. After the initial dose, the remainder of the solution can be injected carefully, and this can be anything from 3 to 5 $\mathrm{ml}$. During the injection or needle placement, paresthesia of the arm and hand may be elicited. If this occurs, it indicates that the needle was lying deep to the tubercle and has been touching the $\mathrm{C} 6$ or $\mathrm{C} 7$ nerve root. Withdrawal and repositioning of the needle are important, repeating the same process of aspiration and repeat of the test dose.

A modification of this technique includes injection at a lower level from the cricoid cartilage so that the tip of the needle would be at the C7 level. However, as the C7 vertebra has no anterior tubercle, palpation is difficult and injury to the pleura and to the vertebral artery is common, especially with the blind technique. (2, 29-30).

The same techniques can be used with fluoroscopy-, MRIor ultrasound-guided technique. The latter techniques require only a minimal volume of local anesthetic agent, ie, 1 to $2 \mathrm{ml}$ compared to $5 \mathrm{ml}$ needed to produce stellate ganglia block or sympathetic blockade to the face, head and neck. Ten milliliters of local anesthetic agent may be required to produce sympathetic blockade to the upper extremity, as the solution should spread down to the T2 / T3 level to block the sympathetic flow to the upper extremity (total sympathetic blockade) $(2,3,15)$.

A continuous technique has been described in which a thin radiopaque Teflon catheter is inserted under local anesthesia and fluoroscopic guidance onto the $\mathrm{C} 7$ (or C6) transverse process. Using the anterior paratracheal technique described above, a 14-gauge cannula (or smaller) can be inserted under local anesthesia at the level of the C6 or C7 transverse process. The stylet is then withdrawn, an epidural catheter can be threaded into the correct position and the original 14-gauge cannula can be removed. It should be recognized, however, that movement of the catheter can still occur in the proximity of the vertebral artery dural cuff and other structures. A small catheter has been used even in pediatric patients (31) since a negative aspiration test cannot rule out migration of the catheter into the vascular structure. A test dose should always be injected or, more preferably, contrast injection under fluoroscopy can be done. The catheter can then be connected to a continuous infusion of a drug to provide continuous sympathetic blockade. This is preferably done for vascular insufficiency or for treatment of postherpetic neuralgia to avoid repeated injections.

\section{CONTRAINDICATIONS}

Contraindications to the procedure include bleeding diathesis, anticoagulant therapy, patient refusal, sepsis at the site of the injection, and bilateral stellate ganglion 
block (or performance of stellate ganglion block in the presence of unilateral recurrent nerve palsy or unilateral phrenic nerve palsy on the opposite side). Performing bilateral stellate ganglion blocks can cause spread and involve the phrenic nerve or the recurrent laryngeal nerve, leading to respiratory embarrassment and the possibility of obstruction of the airway $(2-4,15)$.

\section{SUCCESSFUL SYMPATHETIC BLOCKADE}

Successful sympathetic blockade to the head and neck structures can be easy to recognize clinically and documented by the presence of Horner's syndrome, which includes myosis (pinpoint pupil), ptosis (dropping of the upper eyelid) enophthalmos; also associated with that are conjunctival injection, nasal congestion and facial anhydrosis. However, the presence of Horner's syndrome does not indicate complete interruption of the sympathetic flow to the upper extremity. To test the sympathetic blockade to the upper extremities, multiple methods have been used. Clinical examination, which includes dilatation of the veins in the upper extremity, is inconclusive $(2,15)$. The most commonly used test is measurement of the temperature of the skin. Elevation of the temperature by 1 to $3^{\circ}$ is typically indicative of sympathetic blockade $(12,29,30$, 32 ). This is usually measured by thermography or contact thermometry (13). However, skin temperature is really a balance between the sympathetic vasoconstriction from norepinephrine release and the gradual dilatation from the release of peptides from nociceptors during adrenergic activity (12). Temperature changes in the field of a blocked peripheral nerve will depend on the combined contribution of these two opposing systems (12). From available information, it is apparent that completeness of sympathetic blockade may depend on the chosen monitored parameter. This means that changes in temperature may not always indicate successful total sympathectomy. If the temperature of the arm is already elevated, then further elevation of the temperature is unlikely to happen (12).

Alternative techniques include measurement of skin-resistance (sympathogalvanic response) (12). Malmqvist et al (29) also measured the skin resistance response and the skin resistance level following sympathetic blockade to determine complete sympathectomy. The skin-resistance response was recorded bilaterally at the same time. Skin-resistance level was recorded, as sympathetic nerve block will lead to increased skin resistance $(12,29)$. Malmqvist et al (29) also performed laser Doppler flowmetric testing to the extremities at the same time of the sympathetic blockade. From this study, Malmqvist et al (29) recommended that successful sympathetic blockade should satisfy five criteria, which include Horner's syndrome within 300 seconds; final skin temperature of $34^{\circ} \mathrm{C}$ or more, assuming an original temperature (preblock) of $32^{\circ}$ or lower, ie, 1 to $3^{\circ} \mathrm{C}$ temperature increase; an increase in blood flow by $50 \%$ or more from the preblock status; abolition of skin-resistant response on the radial and ulnar side following the block; and an increase in the skin-resistant response amounting to $13 \%$ or more of the preblock value on the radial and ulnar side of the blocked extremity $(12,29,33)$. Other methods used to predict complete sympathetic blockade include pulse-amplitude changes, which are difficult to quantify (34). Lindberg and Wallin (35) used microneurography, which is a direct but invasive test requiring elaborate equipment and experience $(12,13)$. The sweat test (ninhydrin, cobalt blue and iodine-starch test) (13) is cumbersome and time consuming and not well accepted by a fair amount of patients and therefore not a widely used. Other methods used to measure blood flow include plethysmography (muscle and skin), xenon-133 and sodium -24 clearance (by the skin and muscle) (13). Electromagnetic flow meters have been used for whole limb blood flow (13).

Out of the tests included above, temperature measurement is probably the most widely used technique to assess complete sympathectomy of the affected site.

\section{SELECTION OF THE LEVEL OF SYMPA- THETIC BLOCK}

Studies by Matsumotos $(30,32)$ and also Malmqvist et al (29) have shown that blockade at the level of the C6 vertebra produced more successful sympathetic blockade to the head and neck with less success of sympathetic blockade to the upper extremities, compared to sympathetic blockade at the level of the $\mathrm{C} 7$ vertebra which produces successful sympathetic blockade of upper extremity (29, 30, 32). More appropriately, a sympathetic blockade at the level of T2/T3 using either the posterior approach or the anterior modified approach by Elias (11) should be more appropriately used for the upper extremity $(9,10$, $13,15)$.

Though any local anesthetic can be used, a long-acting agent is preferable (longer-lasting benefit from the block). This block should be repeated twice using a lower volume of two different local anesthetic agents with variable duration of action to see if the response is comparable 
with the duration of the local anesthetic (before a diagnosis of sympathetically maintained pain can be confirmed).

With the use of a smaller volume ( 1 to $2 \mathrm{~mL}$ ) of local anesthetic, the operator can predict the response more accurately to radio frequency ablation or phenol chemical ablation to the sympathetic ganglia $(7,36)$. Hogan et al (5) have shown that the cervical sympathetic chain is superficial to the paravertebral fascia but deep to the carotid sheath. Therefore, injection at the C6 anterior tubercle level, which occupies a space which is cephalad to the sympathetic chain, is more likely to produce sympathetic block to the head and neck structures. The lower cervical stellate ganglia, however are found posteriorly in the chest against the head of the first rib. Spread of the injected local anesthetic solution to the stellate ganglion from the C6 level injection, therefore, requires that injectate should travel posteriorly upon entering the chest. The injected solution was shown in the study by Hogan et al (5) to take a more anterior route into the chest, thus missing the stellate ganglia block. Hogan et al (5) predicted that the sympathetic blockade is probably because of spread of the injectate to the postganglionic sympathetic nerves from $\mathrm{C} 6 / 7$ roots. Injection at the level of the transverse process of $\mathrm{C} 7$, which has no anterior tubercle, means that the plane of the injectate solution would be in the level of the brachial plexus or posterior to it. Injecting at this level makes it undesirable for selective sympathetic blockade, being that the solution is actually spreading anterior to the stellate ganglia, which makes sympathetic block via blocking the stellate ganglia unlikely. Hogan and colleagues $(4,5)$ have explained that stellate ganglia block may actually work by producing postganglionic block of the sympathetic fiber in the brachial plexus rather than the ganglia itself. Another possibility includes blockade of the postganglionic sympathetic fibers along the vertebral and subclavian artery (and with their branches), and also the postganglionic fibers that accompany the brachial plexus, as these structures are positioned more anterior to the stellate ganglia and in the path of the injected local anesthetic agent.

This is vital, as it will explain why using a larger volume can misdirect the purpose of the injection. Precise injection at the level of the stellate ganglia/lower cervical ganglia using either fluoroscopy- or MRI- guided technique is thus vital. The use of a smaller volume of local anesthetic could reduce the spread of local anesthetic and provide additional information about the sympathetic pathway in each patient $(29,36)$. Using a smaller volume of local anesthetic can give an idea as to the degree of sym- pathetic contribution of the upper extremity by the nerve of Kuntz and response to radiofrequency lesion (36).

\section{COMPLICATIONS}

Complications of stellate ganglion block can be divided into technical, infectious and pharmacological.

\section{Technical Complications}

Technical complications include injury to the nerves and nearby viscera during insertion of the needle $(2,12,13$, 15). This includes injury to the brachial plexus; trauma to the trachea and esophagus (with mediastinal and surgical emphysema); injury to the pleura and lung (pneumothorax, hemothorax, which may require chest tube insertion); and bleeding and local hematoma, especially if the patient was taking anticoagulants. This can lead to airway compression (31). Vasovagal attacks can also occur.

\section{Infectious Complications}

Infectious complications are possible if there was a breach in the aseptic barrier. These can include local abscess, cellulitis and osteitis of the vertebral body and transverse process (14).

\section{Pharmacological Complications}

These are related to the dose, volume, type of local anesthetic and site of deposition of the solution. This includes hoarseness of voice because of involvement of recurrent laryngeal nerve or phrenic nerve paralysis, which leads to respiratory embarrassment, especially if there is contralateral dysfunction of the phrenic nerve, or in patients with respiratory dysfunction (this is why bilateral stellate ganglia block is contraindicated) $(2,13,15)$.

Involvement of the brachial plexus may lead to subclinical somatic blockade, which may produce the false impression that the pain is sympathetically maintained. Intra-arterial injection into the vertebral artery or the carotid artery can produce a high concentration of local anesthetic agent in the CNS, leading to seizures. Intravenous injection can lead to seizure, but this is unlikely because of the low volume/dose of local anesthetic (13). Selective nerve-root spread has also been documented; epidurally of the local anesthetic; and intrathecal injection of the local anesthetic producing high spinal blockade has been reported. Horner's syndrome is the final 
consequence of sympathetic blockade, which is really not a complication although it can be unsightly. Air embolism has also been reported. Loss of cardioaccelerator activity may lead to various bradyarrhythmias and hypotension (13).

\section{PERMANENT LESION TO THE LOWER CERVI- CAL SYMPATHETIC/STELLATE GANGLION}

Permanent blockade of the lower cervical sympathetic plexus can be performed in patients with a chronic vascular condition or sometimes in cases where there is sympathetically maintained pain which has responded favorably to repeated sympathetic blockade. Permanent blockade can also be performed in cases of recurrence of the same pathology that have responded initially to sympathetic blockade. In such conditions a permanent lesion to the cervical sympathetic plexus may be indicated.

Multiple techniques for stellate/lower cervical sympathectomy have been suggested. These include open surgical technique, and endoscopic thoracic sympathectomy $(9,10)$.

Multiple nonsurgical techniques for chronic sympathectomy have been described. These include either thermal or lytic lesions. Radiofrequency lesion produces a thermal lesion to the neural structure. Electrical impedance in the surrounding tissue will generate heat in the tissue itself around the electrode through which radiofrequency current has been passed. The tip of the electrode located in the heated tissue will absorb the heat and so can be used to measure the final temperature $(9,10,28,37,38)$. Thermal equilibrium will be reached within 1 to 2 minutes and this is usually the time required to produce the lesion. This produces a discrete lesion. Multiple lesions are required to produce a wider sympathectomy. However a small lesion will avoid injury to the nearby vital structures, including the vertebral artery, carotid artery, the brachial plexus or the recurrent laryngeal nerve. The final lesion depends on multiple factors, mostly the size, shape and configuration of the electrode and the temperature which is used during the equilibrium phase. However, using a temperature of more than $90^{\circ}$ can result in sterile abscess and boiling of the nearby tissue and should be avoided $(9-11,28,38)$. Most authors recommend a temperature between $80^{\circ}$ to $90^{\circ}$ Celsius for the lesion $(9$, $10,28,38)$. The temperature, shape and position of the electrode tip are the most important parameters in producing the lesion $(39,41)$. However other parameters should be measured. These include the power of the cur- rent, voltage and impedance (28). Should any of the values of the above be outside the expected range, performance of the procedure should be stopped and one should look for a short circuit, misplacement of the electrodes and/or an open circuit $(28,38)$.

Other factors that may affect the size of the radiofrequency lesion include nonhomogenous tissue or the presence of heat sinks; which includes blood vessel, CSF or bone (28, 38). Racz et al $(39,41)$ has also recommended the use of a curved needle tip so that a wider lesion can be produced without reinsertion of the needle but with only a $360^{\circ}$ rotation of the needle.

Two types of radiofrequency percutaneous ablation lesion of the stellate/lower cervical sympathetic block have been described. Geurts and Stolker (36) described advancement of a radiofrequency cannula with a $5 \mathrm{~mm}$ active tip as described in the anterior approach until the superior lateral aspect of the $\mathrm{C} 7$ vertebral body is encountered under flouroscopic guidance. The cannula is pulled back anteriorly approximately 2 to $3 \mathrm{~mL}$ to make sure that the active tip is anterior to the longus colli muscle. The final position is on the vertebral body at the junction with the transverse process; proper stimulation techniques are crucial to avoid injury to the phrenic and recurrent laryngeal nerve. A total of three lesions should be made. These include the point as described above, a point just lateral and caudal (on the medial aspect of the transverse process of $\mathrm{C} 7$ ), and finally a lesion at $.1 \mathrm{~cm}$ caudal (on the anterior lateral aspect of the vertebral body of (7).

These three lesions would create a triangle zone of thermal interruption of the cervical sympathetic fibers. Prior to each lesion, electrical stimulation technique should be performed. This includes using $2-\mathrm{V}$ at $50-\mathrm{Hz}$ and $100-$ $\mathrm{Hz}$ stimulation. Using this voltage, there should be no tingling sensation into the upper extremities. At $2-\mathrm{Hz}$ the patient is also asked to say, $E E E$; while stimulating at $2.5-\mathrm{V}$, there should be no impairment of the patient's ability to articulate the letter $E$ continuously. If there is impairment, the cannula is too close to the recurrent laryngeal nerve. At the same time attention should be paid to the rib cage to see if there is movement of the diaphragm; some even recommend placement of a hand just under the rib cage to feel for movement of the diaphragm. Movement of the diaphragm at 2-Hz stimulation means that the needle is in the proximity of the phrenic nerve, although the phrenic nerve should be well lateral to the lesion site; any movement of the diaphragm with $2.5-\mathrm{V}$ at $2-\mathrm{Hz}$ warrants immediate investigation and probably 
repositioning of the cannula. Once the proper stimulation parameters have been met, contrast dye should be injected to confirm no intravascular, epidural or intrathecal spread; then $.5 \mathrm{~mL}$ of $0.25 \%$ bupivacaine or $0.5 \%$ bupivacaine is injected through the cannula. After waiting for 1 to 2 minutes, a lesion is then made at $80^{\circ} \mathrm{C}$ for 30 to 60 seconds. The cannula is immediately moved and the entire process is repeated for the second and third lesions at the sites described above.

Bupivacaine is chosen for its slow onset, which makes it more difficult to inadvertently anesthetize the phrenic or recurrent laryngeal nerve prior to the lesion process. If the canula with a 7-or 10-mm radiofrequency ablation tip is used, the canula can be applied directly to the bone and there is no need to pull the needle back. The longus colli muscle is approximately $5 \mathrm{~mm}$ thick and at its thickest point permits a lesion of at least $2 \mathrm{~mm}$. The lesion on the medial aspect of the $\mathrm{C} 7$ transverse process must be done with extreme care $(3,36)$. The anterior portion of the transverse process is quite narrow at this point and a radiofrequency cannula must stay in the same plane as for the ventral aspect of the vertebral body to prevent injury to the segmental nerve or vertebral artery. Lastly, Kline $(28,38)$ has also advocated an additional lesion in such way that the radiofrequency cannula is directed caudally to the groove where the head of the first rib meets the ventral lateral aspect of the body of T1 vertebra (38). At this ventrolateral position, a fourth lesion can be made to interrupt some of the thoracic sympathetic fibers. This can provide a much wider sympathetic blockade, especially to the upper extremity. Geurts and Stolker (36) have reviewed 27 cases selected out of 40 patients with upper-extremity reflex sympathetic dystrophy (CRPS type 1) in whom they performed radiofrequency lesion to the stellate ganglia. After 6 to 8 weeks' follow-up, 21 patients out of the 27 were pain free, while 4 reported slight improvement in the pain, thus allowing them to participate more with physical therapy. Only two patients reported no benefits. During the follow-up period of 13.2 months (range, 5 to 38 months), 16 patients were still pain free without other signs of reflex sympathetic dystrophy, while 9 have recurrent symptoms. In seven of these cases there appeared to be an underlying triggering factor, for which six required surgery. This makes it clear that, before such a lesion is performed, it is important to appropriately select the patient to be sure that no surgically or medically treatable disease should be addressed. After the 43 radio-frequency lesions produced by this author, there were four initial technical failures, three mi- nor complications and one major complication due to irritation of the phrenic nerve, which resolved after 2 weeks. One of the important messages of this article is that radiofrequency lesions should only be performed in selected patients. The author also recommends that radiofrequency lesion at the $\mathrm{C} 7$ is not indicated if the patient reacts favorably to a diagnostic block of the sympathetic chain at $\mathrm{C} 7$ with a large volume of local anesthetic agent (6 to $10 \mathrm{~mL}$ ), resulting in Horner's syndrome and painless, warm, dry hand but fails to react to an injection of only $1 \mathrm{~mL}$ of local anesthetic agent deposited at the same area, ie, at the junction of the transverse process and the vertebral body of $\mathrm{C} 7$, despite the signs of adequate sympathetic blockade to the hand. The author attributes the improvement when the larger volume is used to the blockade of the T2/T3 sympathetic ganglia (overflow of the local anesthetic). These two ganglia are out of reach of a lesion produced by the radiofrequency ablation at the level of the $\mathrm{C} 7$ vertebra. Another explanation is that the use of a large volume (6 to $10 \mathrm{~mL}$ ) of local anesthetic agent may have produced benefit not because of the blockade of the sympathetic ganglia but because of involvement of the somatic nerve because of the spill of the local anesthetic agent to the brachial plexus $(4,5)$. When larger volumes are used, another false-positive reaction may be due to the systemic absorption of the local anesthetic $(4,5)$.

Sluijter (40) has also described another technique. The radiofrequency cannula, either an SMK50 mm or SMK100 mm, is advanced from the anteroposterior view under fluoroscopic guidance until the superior lateral aspect of the C6 body is encountered. A second cannula is passed until the superior aspect of the $\mathrm{C} 7$ vertebra is encountered. Sluijter's (40) technique involves only an extra lesion at the level of C6, again using the same parameters for electrical stimulation and testing produced by Geurts and Stolker (36). He also recommends the injection of a contrast to ensure no epidural/subdural or intravascular spread of the local anesthetic agent. One milliliter of $2 \%$ lidocaine is then injected through each cannula at the $\mathrm{C} 6$ and $\mathrm{C} 7$ and a lesion is created after 1 to 2 minutes at a temperature of $80^{\circ} \mathrm{C}$ for 60 seconds. The lesion produced here is a very discrete lesion and does not interrupt the entire ganglia. Thus, Horner's syndrome is unlikely to be severe. However, impressive results have been reported by Sluijter (40) with this technique. The technique also can be repeated if necessary by repositioning of the needle more medial and lateral to the initial site of insertion. Such lesion of the stellate ganglia can be used for treatment of any type of sympathetically main- 
tained pain in the upper extremity.

An injection of 1 or $2 \mathrm{~mL}$ of $6 \%$ aqueous phenol or $10 \%$ phenol at the level of $\mathrm{C} 7$ or $\mathrm{C} 6$ has been used to produce a chemical sympathectomy. This technique is not commonly practiced because of the proximity of the somatic nerves, as it can result in neuritis. The dural sheath may also be entered during the injection. Injection of a solution into the epidural space may lead to a devastating condition; indeed, a case of paraplegia has been reported following upper thoracic sympathectomy using neurolytic agent. Racz (41) has described a relatively safe technique using both phenol $3 \%$ and a mixture of $6 \%$ phenol with $.5 \%$ plain bupivacaine. There have been favorable reports regarding the efficiency of this procedure, as well as the morbidity and mortality. The advantage of using neurolytic technique is that it covers more of the sympathetic fibers and hence produces a broader lesion and a more complete sympathectomy. The disadvantage of this method is that it is more difficult to control the lesion. The radiofrequency lesion is well controlled and there can be no spread of the lesion outside the 5-mm tip radius.

Complications of these techniques are similar to the complications produced by local anesthetic sympathetic ganglia block, with the exception of a longer lasting block and potential neuritis.

\section{POSTSYMPATHECTOMY SYNDROME}

Post sympathectomy neuralgia is a poorly understood painful condition, which occurs in up to $50 \%$ of all patients undergoing sympathectomy for the treatment of chronic pain (42). This also includes patients undergoing cervical/thoracic sympathectomy. Post sympathectomy neuralgia is proposed to be a complex neuropathic and central reafferentation and deafferentation syndrome. It depends on the transection during sympathectomy of the paraspinal somatic and visceral afferent axons, which are transmitted within the sympathetic trunk. Subsequently, the cell death of many of the nociceptive axotomized afferent neurons will result in central deafferentation and persistent sensitization of the spinal nociceptive neurons by the painful condition already present prior to the sympathectomy (viscerosomatic conversions and collateral sprouting of afferent nerves). The mechanics associated with sympathetically maintained pain are all proposed to be important factors in the development of this syndrome. This syndrome can appear anywhere from a few days to a few weeks following chemical or surgical sympathectomy.
It is characterized by deep, aching pain with superficial burning and hyperesthesia, which may or may not respond to narcotic analgesic. Post sympathectomy neuralgia is usually localized to the proximal region of the sympathectomized limb and to the trunk. This is sometimes confused with recurrence of CRPS type I or type II or the original pathology which led to the performance of the sympathectomy. It is interesting that this postsympathectomy neuralgia is also sympathetically maintained. This is explained by the fact that, if this occurs following sympathectomy for hyperhidrosis, it only occurs in the area where there is recurrence of the sweating. This syndrome has been reported in $30 \%$ to $50 \%$ of all sympathectomized patients. It is interesting that it was only reported in $6 \%$ of patients who have undergone sympathectomy for hyperhidrosis. This is in distinct contrast to the $40 \%$ to $50 \%$ incidence of post sympathectomy neuralgia following sympathectomy for CRPS type I and type II.

Bonica (15) has indicated that cardiac, esophageal and tracheobronchial afferents that project to their target via the sympathetic trunk are removed during cervical sympathectomy, ie, the lower part of C8/T1 (stellate ganglia) and the T1/T3 ganglia that are sometimes removed for upper-extremity sympathetically maintained pain (visceral somatic conversions theory). Transection of these nerves during the $\mathrm{C} 8$ /T1 or even T2/ T3 sympathectomy can result in somatic referral of pain to the trunk and the proximal limb as the result of this visceral sympathetic afferent activity (deafferentation with the developing of firing ectopic neuron or because of central hypersensitization). The end result is likely to be referral of pain to the somatic tissue of the trunk and the proximal limb (42). It has also been shown that some paraspinal somatic afferent nerves do project to their ventral spinal target tissue via the lumbar sympathetic trunk. Clearly, these nerves will be damaged during sympathectomy. This can lead to central deafferentation and central sensitization with aggravation of the pain syndrome. As we understand the CNS more and more, there are multiple factors which can aggravate this condition. They include extensive convergence of nerves within the spinal cord. This pain syndrome post sympathectomy neuralgia may even involve a wider area of the body, including almost half of the body on the same side of the lesion. This can be mistaken as spread of the reflex sympathetic dystrophy.

Treatment of post sympathectomy neuralgia depends on the symptoms. If it is due to firing, spontaneous pain, then phenytoin, carbamazepine or gabapentin can be help- 
ful. Mexiletine or lidocaine given IV can also be helpful in cases where there is spontaneous pain or allodynia. Tricyclic antidepressants can also be helpful to reduce the incidence of post sympathectomy neuralgia. A discreet sympathetectomy should be produced. However, it is unavoidable that some transection of the paraspinal somatic afferent axon will occur during sympathectomy procedures. Post sympathectomy neuralgia develops more frequently if pain is the predominant picture of the disease that requires the sympathectomy. Use of pre-emptive analgesia technique, ie, continuous epidural or continuous stellate ganglia block or cervical block, can reduce the barrage of nociceptive stimulation to the CNS; this may help reduce postsympathectomy syndrome. During surgical sympathectomy, post sympathectomy neuralgia could be avoided if the proximal stump of the transected nerve is enclosed in an implantable sheath. This has been suggested to prevent centripetal transport of neurotoxic cells released from the injured tissue of the locus of the transection. And, finally, repeating the sympathetic blockade and the sympathectomy with more complete sympathectomy can also relieve this syndrome. It has also been suggested that spinal cord stimulation or dorsal column stimulation can be of little help with this syndrome, as one of the mechanisms of action of spinal cord stimulation is through the sympathetic nervous system. The fact that these patients have already had a damaged or interrupted sympathetic system may mean that they would respond poorly to a spinal cord stimulation trial. Indeed, some authors have recommended that no sympathectomy be used for treatment of CRPS type I and type II until a trial of spinal cord stimulator has been attempted.

\section{CONCLUSION}

Cervical sympathetic blockade/stellate ganglia blockade is a very commonly performed procedure. If performed correctly, this can provide good therapeutic, prognostic and diagnostic values. It is vital that these procedures should be done under fluoroscopic guidance. Using blind technique, with the proximity of these sympathetic chains to a nearby vascular structure and neural structure, not only makes the diagnosis inappropriate because of spill to the somatic nerve but also makes it dangerous because of injury to nearby structures. It is recommended that only $1 \mathrm{~mL}$ of solution should be used for diagnostic or prognostic stellate ganglia block to predict the response to radiofrequency ablation/chemical sympathectomy to the cervical sympathetic ganglia. Using a larger volume can confuse the outcome and prognosis of the block. However, for therapeutic purposes, ie, only for the relief of pain during performance of physical therapy or rehabilitation or to improve the circulation to an infected limb, a larger volume of local anesthetic can be used (solely for therapeutic purposes). However, the diagnostic and prognostic benefits of such block will be lost. These procedures should be performed while monitoring the temperature and the skin blood flow to the upper extremities, as the presence of Horner's syndrome does not guarantee a complete sympathectomy to the upper extremities. Stellate ganglia block can produce a total sympathectomy to the head and neck structure. However, it will produce an incomplete sympathetic blockade to the upper extremities and a combination of stellate/upper cervical plus lower cervical, ie, T2/T3 sympathectomy, is required to produce complete sympathectomy to the upper extremities.

\section{REFERENCES}

1. Ellis H, Feldman S. Anatomy for the anaesthetists, ed 3. Oxford, Blackwell Scientific Publications, 1979; pp256-262.

2. Buckley FP, MorriccaG, Murphy TM. Neurolytic blockade and hypophysectomy. In: Bonica JJ (ed). The Management of Pain, ed 2. Philadelphia, Lea and Febiger, Vol. II, 1990; pp2012-2014.

3. Raj.PP. Stellate ganglion block. In: Waldman SD, Winne AP (eds). Interventional Pain Management. Philadelphia, Dannemiller Education, WB Saunders Company, 1996.

4. Hogan Q, Erickson SJ. MR imaging of the stellate ganglion; Normal appearance. AJR 1992; 158:655659 .

5. Hogan Q, Erickson S, Haddox D et al. The spread of solution during stellate ganglion block. Regional Anesthesia 1992; 17:78-83.

6. Guntmukkala M, Hardy A. Spread of injectate after stellate ganglion block in man: An anatomical study. BJA 1991; 66:643-644.

7. Slapppendel F, Thijssen H, Crul BJ et al. The stellate ganglion in magnetic resonance imaging, a quantification of anatomic variability. Anesthesiology 1995; 83:424-426.

8. Christie J, Martinez C. Computerized axial tomography to define the distribution of solution after stellate ganglion block. J of Clinical Anesthesia 1995; 7:308311.

9. Wilkinson H. Percutaneous radiofrequency, upper thoracic sympathectomy. New technique. Neurosurgery $1984 ; 15: 811-814$.

10. Wilkinson H. Neurosurgical procedures of the sympathetic nervous system. Pain Clinic 1995; 1:43-50.

11. Elias M. The anterior approach for thoracic symphatetic ganglion block using a curved needle. Pain Clinic 2000; 12:17-24. 
12. Hogan Q, Abram S. Neural blockade for diagnosis and prognosis. Anesthesiology 1997; 86:216-241.

13. Lofstrom B, Cousins M. Sympathetic neural blockade of the upper and lower extremity. In Neural Blockade in Clinical Anesthesia and Management of Pain, ed 2. Cousins M, Bridenbaugh PO (eds). London, J.P. Lippincot, 1988.

14. Hartzler GO, Osborn MJ. Invasive electrophysiological study in the Jervell and Lange-Nielsen syndrome. Br Heart J 1981; 45:225-229.

15. Bonica JJ. Sympathetic nerve blocks for pain diagnosis and therapy. Technical considerations vol. II. Block of stellate ganglion and cervicothoracic and thoracic chains. Published by Winthrop - Breon laboratories 1984; pp61-77.

16. Price DD, Long S, Rafi A. Analysis of peak magnitude and duration of analgesia produced by anesthetic injection into sympathetic ganglion in complex regional pain syndrome patients. Clin J Pain 1998; 14:216-226.

17. Wassef MR. Phantom pain with probable reflex sympathetic dystrophy; Efficacy of fentanyl infiltration of the stellate ganglion. Reg Anesth 1997; 22:287-290.

18. Yanagita N, Innafuku S, Suzuki Y. Stellate ganglion block for perceptive tinnitus. Jibinkoka 1971; 43:989999.

19. Cook TG, de Sanctis CA, Plaza JA et al. Stellate ganglion block for sudden profound hearing loss. $A n$ esthesiology 1981; 54:421-423.

20. Ohinata Y, Makimoto K, Kawakami M, et al. Blood flow in the common carotid and vertebral arteries in patients with sudden deafness. Am Otol Rhinol Laryngol 1997; 106:27-32.

21. Haug O, Draper WL, Haug SA. Stellate ganglion block for sudden idiopathic sensorineural hearing loss. Arch Otolaryngol 1976; 102:5-8.

22. Yanagida H, Kemi C, Suwa K. The effects of stellate ganglion block on idiopathic prolongation of the Q-T interval (the Romano-Ward syndrome). Anesth Analg 1976; 55:782-787.

23. Lynch ME, Elgeneidy AK. The role of sympathetic activity in neuropathic orofacial pain. J Orofac Pain 1996; 10:197-305.

24. Saxen MA, Campbell RL. An unusual cause for sympathetically maintained facial pain complicating telangiectasia. Oral Surg Oral Med Oral Radiol Endod 1995; 79:455-458.

25. Arden RL, Bahu SJ, Zuazu MA et al. Reflex sympathetic dystrophy of the face; Current treatment recommendation. Laryngoscope 1998; 108:437-442.

26. Lehmann LJ, Warfield, Bajwa ZII. Migraine headache following stellate ganglion block for reflex sympathetic dystrophy. Headache 1996; 36:335-337.

27. Kapral S, Krafft P, Gosch M et al. Ultrasound imag- ing for the stellate ganglion block; Direct visualization puncture site and local anesthetic spread. A pilot study. Reg Anesth 1995; 20:323-328.

28. Kline M. Radiofrequency lesion of the stellate ganglion. Stereotactic radiofrequency lesion as part of the management of pain. St.Lucie Press. Radionics, Inc, 1996.

29. Malmqvist EL, Bengtsson M, Sorenson J. Efficacy of stellate ganglion block: A clinical study with bupivacaine. Reg Anesth 1992; 17:340-347.

30. Matsumoto $S$. Thermographic assessments of the sympathetic blockade by stellate ganglion (1); Comparison between C7-SGB and C6-SGB in patients. Masui 1991; 40:562-569.

31. Elias M, Chakerian M. Repeated stellate ganglion block using a catheter for pediatric herpes zoster ophthalmicus. Anesthesiology 1994; 80:950-952.

32. Mastsumoto S. Thermographic assessment of the sympathetic blockade by stellate ganglion block (20); Comparison between C7-SGB and C6-SGB in 20 healthy volunteers. Masui 1991; 40:692-701.

33. Stevens RA, Stotz A, Kao TC et al. The relative increase in the skin temperature after stellatae ganglion block is predictive of complete sympathectomy of the hand. Reg Anesth Pain Med 1998; 23:266-270.

34. Meijer J, deLange J, Ros H. Skin pulse wave monitoring during lumber epidural and spinal anesthesia. Anesth Analg 1988; 67:356-359.

35. Lindenberg L, Wallin BG. Sympathetic skin nerve discharges in relation to amplitude of skin resistance response. Psychophysiology 1981; 18:268-270.

36. Geurts JW, Stolker RJ. Percutaneous radiofrequency lesion of the stellate ganglion in the treatment of pain in upper extremity reflex sympathetic dystrophy. Pain Clinic 1993; 6:17-25.

37. Vervest AC, Stolker RJ, Groen GJ. Radiofrequency lesioning for pain treatment. Pain Clinic 1995; 8:175189.

38. Kline M. Radiofrequency techniques in clinical practice Part III. In: Waldman SD, Winne AP (eds). Interventional Pain Management. Philadelphia, Dannemiller Memorial Education Foundation ,W.B. Saunders Company, 1996; p185.

39. Racz G, Finch P, McDaniel K. A curved approach to nerve blocks and radiofrequency lesioning. Pain $\mathrm{Di}$ gest 1997; 7:251-257.

40. Sluijter M. Radiofrequency Lesion on the Treatment of Cervical Pain Syndrome. Boston, Radionics Corp, 1990.

41. Racz G (ed). Techniques of Neurolysis. Boston, .Kluwer Academic Publications, 1989; pp99-124.

42. Kramis RC, Roberts WJ, Gillette RG. Post-sympathectomy neuralgia: Hypotheses on peripheral and central neural mechanisms. Pain 1996; 64:1-9. 